\title{
ON FROBENIUS EXTENSIONS I.
}

\author{
TADASI NAKAYAMA and TOSIRO TSUZUKU
}

As a generalization of the notion of Frobenius algebras over a field Kasch [10] introduced that of Frobenius extensions of a ring. The present writers [13] recently freed one of Kasch's main theorems from its rather strong $S$-ring assumption of the ground ring. However, even with the removal of the $S$-ring assumption of the ground ring the notion does not seem general enough, and we wish, in the present paper and its sequel, to develope the theory upon the basis of a more general notion of Frobenius extensions. Thus, we replace the free module property of the extension by the projective module property (according to a general tendency in algebra), which has been done in fact in case of Frobenius algebras over a commutative ring in a previous work by Eilenberg and one of the writers [4], and, further, take automorphisms of the ground ring $^{1)}$ into the definition of Frobenius extensions (which seems quite natural particularly in case of non-commutative rings). To such generalized notion of Frobenius extensions we may extend many of Kasch's theorems, including those which are immediate extensions of classical theorems for Frobenius algebras and those which are essentially new, as the above alluded endomorphism ring theorem. Also homological properties of Frobenius extensions, as were developed in Hirata's [6] recent paper in succession to Eilenberg-Nakayama [4], can be extended to our present generalized case; we shall also exceed [4], [6] somewhat in considering injective and weak dimensions.

On the other hand, we restrict ourselves, in the present work, to extensions which are finitely generated modules over a ground ring. It is perhaps appropriate to treat "infinite" Frobenius extensions in another work, since with them we would face many problems of new kinds as Jan's [9] recent work on infinite Frobenius algebras already shows.

\section{$\S 1$. Frobenius extensions}

In the present work a ring shall always have a unit element 1 and its

Received April 15, 1960.

1) Cf. a remark at the end of $\$ 1$. 
subring shall contain 1 . If $\mathfrak{M}, \mathfrak{R}$ are right (say)-modules over a ring $B$ (which we express by writing $\mathfrak{M}_{B}, \mathfrak{N}_{B}$ ) and if $\beta$ is an automorphism of $B$, we denote by $\operatorname{Hom}_{B, \beta}(\mathfrak{M}, \mathfrak{R})$, or $\operatorname{Hom}_{B, \beta}\left(\mathfrak{M}_{B}, \mathfrak{N}_{B}\right)$, the module of all $(B, \beta)$-homomorphisms of $\mathfrak{M}$ into $\mathfrak{N}$, i.e. homomorphisms $\varphi$ (for mere additive groups) of $\mathfrak{M}$ into $\mathfrak{R}$ satisfying $\varphi(u b)=(\varphi u) \beta b$ for all $u \in \mathfrak{M}, b \in B$. In case of $A$-B-module $A_{A} \mathfrak{M}_{B}$ the module $\operatorname{Hom}_{B, \beta}\left(\mathfrak{N}_{B}, \mathfrak{N}_{B}\right)$ has an $A$-right-module structure defined by $\left.{ }^{2}\right)(\varphi a) u$ $=\varphi$ au $(a \in A)$, as usual, while in case of $C$-B-module $c \Re_{B}$ it has a $C$-left-module structure defined by $(c \varphi) u=c(\varphi u) \quad(c \in C)$.

Proposition 1. Let $A$ be a ring and $B$ a subring of $A$. The set of conditions :

$\left.1_{r}\right)$ the B-right-module $A$ is finitely generated and projective,

$\left.2_{r}\right)_{B} A_{A} \approx \operatorname{Hom}_{B, \beta}\left(A_{B}, B_{B}\right)$ (B-left, A-right isomorphism) with some automorphism $\beta$ of $B$,

is equivalent to the set of conditions:

$\left.1_{l}\right)$ the $B$-left-module $A$ is finitely generated and projective,

$\left.2_{l}\right)_{A} A_{B} \approx \operatorname{Hom}_{B, \beta^{\prime}}\left({ }_{B} A,{ }_{B} B\right)$ (A-left, B-right isomorphism) with some automorphism $\beta^{\prime}$ of $B$.

Proof. Assume $1_{r}$ ). As $A_{B}$ is then a direct summand of a finitely generated free $B$-right-module, it follows readily that the $B$-left-module $\operatorname{Hom}_{B, \beta}\left(A_{B}\right.$, $B_{B}$ ) is finitely generated and projective. So, if we further assume $2_{r}$ ), we obtain $1_{l}$ ).

Next, generally (i.e. without assuming $\left.1_{r}\right), 2_{r}$ )) we obtain an $A$-left, $B$-right homomorphism

$$
\begin{aligned}
& \tau:{ }_{A} A_{B} \rightarrow \operatorname{Hom}_{B, \beta-1}\left(\operatorname{Hom}_{B, \beta}\left(A_{B}, B_{B}\right),{ }_{B} B\right) \quad \text { by defining } \\
& (\tau a) \varphi=\beta^{-1} \varphi a\left(a \in A, \varphi \in \operatorname{Hom}_{B, \beta}\left(A_{B}, B_{B}\right)\right) ;
\end{aligned}
$$

that $\tau a$ is a $\left(B, \beta^{-1}\right)$-homomorphism of $\operatorname{Hom}_{B, \beta}\left(A_{E}, B_{B}\right)$ into ${ }_{B} B$ may be seen in

$$
\begin{aligned}
(\tau a) b \varphi & =\beta^{-1}(b \varphi) a=\beta^{-1} b \varphi a=\left(\beta^{-1} b\right) \beta^{-1} \varphi a \\
& =\left(\beta^{-1} b\right)(\tau a) \varphi \quad(b \in B)
\end{aligned}
$$

and the $A$-left, $B$-right homomorphism property of $\tau$ may be seen also readily

2) $\varphi$ au means $\varphi(a u)$. This principle on the order of operations will be adopted throughout the present paper, if some parentheses or other indications or limitations do not point out or restrict otherwise. 
in $^{3)}$

$$
\begin{aligned}
\left(\tau\left(a_{1} a\right)\right) \varphi & =\beta^{-1} \varphi\left(a_{1} a\right)=\beta^{-1} \varphi^{a 1} a=(\tau a) \varphi^{a_{1}} \\
& =\left(a_{1} \tau a\right) \varphi \quad\left(a_{1} \in A\right) \\
(\tau(a b)) \varphi & =\beta^{-1} \varphi(a b)=\beta^{-1}(\varphi a) \beta b=\left(\beta^{-1} \varphi a\right) b \\
& =((\tau a) \varphi) b=((\tau a) b) \varphi
\end{aligned}
$$

If $A$ is $B$-right-projective, then $\tau$ is monomorphic, since in that case there exists for every $a \neq 0$ in $A$ a $\varphi$ with $\varphi a \neq 0$ as we readily see by imbedding $A_{B}$ into a free $B$-right-module. If further $1_{r}$ ) is assumed, then $\tau$ is epimorphic too, as we see easily on considering $A_{B}$ as a direct summand of a finitely generated free $B$-right-module. So, if $1_{r}$ ) and $2_{r}$ ) are assumed, we have

$$
\begin{aligned}
{ }_{A} A_{B} & \approx \operatorname{Hom}_{B, \beta^{-1}}\left(\operatorname{Hom}_{B, \beta}\left(A_{B}, B_{B}\right),{ }_{B} B\right) \\
& \approx \operatorname{Hom}_{B, \beta^{-1}}\left({ }_{B} A,{ }_{B} B\right) \quad(A \text {-left, } B \text {-right-isomorphisms }),
\end{aligned}
$$

proving $2_{l}$ ) with $\beta^{\prime}=\beta^{-1}$.

By symmetry $1_{l}$ ) and $2_{l}$ ) imply $1_{r}$ ) and $2_{r}$ ) (with $\beta=\beta^{\prime-1}$ ).

Now we introduce

Definition. A ring $A$ is said to be a 2. Frobenius extension (or a Frobenius extension of 2 nd kind) of its subring $B$ when the equivalent sets of conditions $\left.1_{r}\right), 2_{r}$ ) and $1_{l}$ ), $2_{l}$ ) are fulfilled. If we wish to make the automorphism $\beta$ in $2 r$ ) explicit, we speak of a 2 . Frobenius extension with respect to $\beta$, or simply a $\beta$-Frobenius extension. Further, a 1-Frobenius extension is called a 1. Frobenius extension (or a Frobenius extension of 1st kind).

Remark. The condition $2 r)$ implies that the $A$-right-module $A$ is $(A, B)$ relatively injective (not only projective, trivially), in the sense of Hochschild [7]; cf. Lemma 1 there. Symmetrically, a 2. Frobenius extension $A$ of $B$ is $(A, B)$-relatively left injective.

Proposition 2. Let $A$ be a $\beta$-Frobenius extension of $B$, and $\beta^{\prime}$ be a second automorphism of $B$. Then $A$ is a $\beta^{\prime}$-Frobenius extension of $B$ if and only if the automorphism $\beta^{\prime} \beta^{-1}$ of $B$ is induced by an inner automorphism of $A$.

Proof. Set $\gamma=\beta^{\prime} \beta^{-1}$. By $\Gamma: \varphi \rightarrow \gamma \varphi$ we obtain evidently a $(B, \gamma)-(A, 1)$ -

3) A right operater is written in exponential form, on the shoulder of an element, whenever there is an ambiguity; indeed, $\varphi_{a_{1} a} a$ would mean $\varphi\left(a_{1} a\right)$. 
isomorphism $\Gamma$ of $\operatorname{Hom}_{B, \beta}\left(A_{B}, B_{B}\right)$ to $\operatorname{Hom}_{R, \beta^{\prime}}\left(A_{B}, B_{B}\right)$. Now, suppose $A$ is simultaneously $\beta$ - and $\beta^{\prime}$-Frobenius over $B$ and let accordingly $\Phi$, $\Phi^{\prime}$ be $B$ - $A$ isomorphisms of ${ }_{B} A_{A}$ to $\operatorname{Hom}_{B, \beta}\left(A_{A}, B_{B}\right), \operatorname{Hom}_{B, \beta^{\prime}}\left(A_{B}, B_{B}\right)$ respectively. The composition $\rho=\Phi^{\prime-1} \Gamma \Phi$ is then a $(B, \gamma)-(A, 1)$-automorphism of ${ }_{B} A_{A}$. Hence $\rho 1$ is a regular element in $A$ (since $(\rho 1) A=\rho A=A$ and $(\rho 1) x=0 \quad(x \in A)$ implies $\rho x=0, x=0$ ), and $\gamma$ is induced by the transformation by $\rho 1$ (since $(\gamma b) \rho 1=\rho b=(\rho 1) b$ for $b \in B)$. The converse can be seen by reversing the argument.

Proposition 3. Let $A$ be a $\beta$-Frobenius extension of $B$, and $\Phi$ be $a B$ - $A$ isomorphism of ${ }_{B} A_{A}$ to $\operatorname{Hom}_{B, \beta}\left(A_{B}, B_{B}\right)$. A second map $\Phi^{\prime}$ of ${ }_{B} A_{A}$ to $\operatorname{Hom}_{B, \beta}$ $\left(A_{B}, B_{B}\right)$ is a $B$-A-isomorphism if and only if $\Phi^{-1} \Phi^{\prime}$ is a left multiplication of a regular element in $A$ element-wise commutative with $B$.

Proof. This is readily seen either directly or by setting $r=1$ (and interchanging $\Phi$ and $\Phi^{\prime}$ ) in the proof to the preceding proposition.

Remark. Proposition 3 indicates already that it will be useful to generalize the notion of Frobenius extensions further by taking (not only 'automorphisms of the subring $B$ but) some automorphisms of the extension ring $A$ and some isomorphisms of $B$ with a second subring of $A$. Postponing our study of such further generalizations till a later paper, we will however restrict ourselves to the above defined 1. and 2. Frobenius extensions in the present paper, since they seem to offer a certain suitable degree of generality.

$\S 2$. Bilinear form and scalar product characterizations of Frobenius extensions

Let $A$ be a 2. Frobenius extension of $B$ with respect to an automorphism $\beta$ of $B$, and let $\Phi$ denote a $B$-A-isomorphism of ${ }_{B} A_{A}$ to $\operatorname{Hom}_{B, \beta}\left(A_{B}, B_{B}\right)$. $\varnothing$ induces an $A \cdot B$-isomorphism $F$ of $\operatorname{Hom}_{B, \beta^{-1}}\left(\operatorname{Hom}_{B, \beta}\left(A_{E}, B_{B}\right),{ }_{B} B\right)$ to $\operatorname{Hom}_{B, \beta^{-1}}$ $\left({ }_{B} A,{ }_{B} B\right)$, which was used in (6). There we proved also that the natural $A-B$ homomorphism $\tau$ of ${ }_{A} A_{B}$ to $\operatorname{Hom}_{B, \beta}-1\left(\operatorname{Hom}_{B, \beta}\left(A_{B}, B_{B}\right),{ }_{B} B\right)$ is an isomorphism, to the effect to obtain in (6) an $A$-B-isomorphism $\Psi$ of ${ }_{A} A_{B}$ to $\operatorname{Hom}_{B, \beta-1}\left({ }_{B} A,{ }_{B} B\right)$ by

$$
\Psi=F \tau \text {. }
$$

This explicit expression of (6) gives a 1-1 correspondence, $\Phi \rightarrow \Psi$, between the 
isomorphisms in $2_{r}$ ) and those in $2_{l}$ ) with $\beta^{-1}=\beta^{\prime}$, since $\tau$ is fixed and since the correspondence can be reversed by symmetry.

We may express the correspondence in a more symmetric form. Thus, we have by (2), (7)

$$
(\Psi a) x=(F \tau a) x=(\tau a)(\Phi x)=\beta^{-1}(\not x) a
$$

for all $a, x \in A$. Further, $(\Psi a) x=(a \Psi 1) x=(\Psi 1)(x a)$ and similarly $(\Phi x) a$ $=\left((\Phi 1)^{x}\right) a=(\Phi 1)(x a)$. Hence

$$
(\Phi 1) x=\beta(\Psi 1) x
$$

for all $x \in A$, or, in other words,

$$
\Phi 1=\beta \Psi 1 .
$$

Proposition 4. If $A$ is $a$ 2. Frobenius extension of $B$ with respect to an automorphism $\beta$ of $B$ and if $\Phi$ is a $B$-A-isomorphism of ${ }_{R} A_{A}$ to $\operatorname{Hom}_{B, \beta}\left(A_{F}, B_{B}\right)$, then $\pi=\emptyset 1$ is $a(B, 1)$-( $B, \beta)$-(bilinear) homomorphism of the $B$-two sided module $A$ into $B$ satisfying

$\left.\mathrm{i}_{r}\right) \pi a A=0(a \in A)$ implies $a=0$,

$\left.\mathrm{i}_{l}\right) \pi A a=0(a \in A)$ implies $a=0$,

$\left.\mathrm{ii}_{r}\right)$ for every $\varphi$ in $\operatorname{Hom}_{B, \beta}\left(A_{B}, B_{B}\right)$ there exists an element a in $A$ with $\varphi x=\pi a x$ $\left(=\pi^{a} x\right)$ for all $x \in A$ (i.e. $\left.\operatorname{Hom}_{B, \beta}\left(A_{B}, B_{B}\right)=\pi^{A}\right)$,

ii $) \operatorname{Hom}_{B, \beta^{-1}}\left({ }_{B} A,{ }_{B} B\right)=A \beta^{-1} \tau$.

Conversely, if $A$ is an extension of $B$ satisfying the condition $1_{r}$ ) (of Proposition $1)$ and if there is $a(B, 1)-(B-\beta)$-homomorphism $\pi$ of ${ }_{B} A_{R}$ into $B$ satisfying $\left.i_{r}\right)$ and $\mathrm{ii}_{r}$ ), where $\beta$ is an automorphism of $B$, then $A$ is a $\beta$-Frobenius extension over $B$ and $\pi=\Phi 1$ with a certain $B$-A-isomorphism $\Phi$ of ${ }_{B} A_{A}$ to $\operatorname{Hom}_{B, \beta}\left(A_{B}, B_{B}\right)$ (and $\pi$ satisfies $\mathrm{i}_{l}$ ), $\mathrm{ii}_{l}$ ) too).

Proof. Under the assumption for the first half, we have $\pi=\emptyset 1=\beta \Psi 1$ (with $\Psi$ as before) and, therefore, $\pi \in \operatorname{Hom}_{B, 1}\left({ }_{B} A,{ }_{B} B\right), \in \operatorname{Hom}_{B, \beta}\left(A_{B}, B_{B}\right)$ and is thus a $(B, 1)-(B, \beta)$-homomorphism of ${ }_{1 ;} A_{B}$ into $B$. If $\pi a A=0$ then $0=(\Phi 1) a A$ $=(\Phi 1)^{a} A=(\Phi a) A$ whence $\Phi a=0$ and so $a=0$. Similarly $\pi A a=0$ implies $a=0$. Further, $\pi^{A}=(\Phi 1)^{A}=\Phi A=\operatorname{Hom}_{B, \beta}\left(A_{B}, B_{B}\right)$. Similarly we have $\left.\mathrm{ii}_{l}\right)$.

Conversely, for any automorphism $\beta$ of $B$ and any $(B, 1)-(B, \beta)$-homomorphism $\pi$ of ${ }_{B} A_{B}$ into $B$ we obtain a $B$-A-homomorphism $\Phi$ of ${ }_{B} A_{A}$ into 
$\operatorname{Hom}_{B, \beta}\left(A_{B}, B_{B}\right)$ on associating each $a \in A$ with $\varphi_{a} \in \operatorname{Hom}_{B, \beta}\left(A_{B}, B_{B}\right)$ defined by $\varphi_{a} x=\pi a x$, i.e. by $\varphi_{a}=\pi^{a}$. When $\mathrm{i}_{r}$ ), $\mathrm{ii}_{r}$ ) are satisfied, $\Phi$ is (a monomorphism and an epimorphism, whence) an isomorphism. Hence $A$ is a $\beta$-Frobenius extension of $B$ provided that $\left.1_{r}\right)$ is satisfied too. We have $\pi x=\varphi_{1} x=\left(\Phi_{1}\right) x$.

Corollary 5. Under $\left.1_{r}\right), A$ is $\beta$-Frobenius over $B$ if and only if there is a $(B, 1)$ - $(B, \beta)$-homomorphism $\pi$ of ${ }_{B} A_{B}$ into $B$ satisfying $\left.\left.\mathrm{i}_{r}\right), \mathrm{ii}_{r}\right)$.

We note also that the existence of a $(B, 1) \cdot(B, \beta)$-homomorphism $\pi$ of ${ }_{B} A_{B}$ into $B$ satisfying $\left.\mathrm{i}_{r}\right), \dot{\mathrm{i}}_{l}$ ) is equivalent to the existence of a $1-\beta$-scalar product $\langle>$ in $B$ of the $B$-left-module $A$ and the $B$-right-module $A$ satisfying

0) $\langle x, y z\rangle=\langle x y, z\rangle$ for $x, y, z \in A$,

i) $\langle>$ is regular.

(A $\beta_{0}-\beta$-scalar product $\langle>$ in $B$ of a $B$-left-module $\mathfrak{L}$ and a $B$-right-module $\Re$ is a $\left(B, \beta_{0}\right)-(B, \beta)$-bilinear map of $\mathfrak{Q} \times \Re$ into $B$, and it is called regular if $\langle x, \mathfrak{R}\rangle=0$ implies $x=0$ and $\langle\mathfrak{R}, y\rangle=0$ implies $y=0$ ). We have merely to set $\langle x, y\rangle=\pi x y$.

Now, with $\pi$ as in Proposition 4, set

$$
\begin{aligned}
& R_{\pi} X=\{x \in A \mid \pi X x=0\}, \\
& L_{\pi} X=\{x \in A \mid \pi x X=0\}
\end{aligned}
$$

for a subset $X$ of $A$. We have

Proposition 6. Let $A$ be a 2. Frobenius extension of $B$ and let $\pi=\emptyset 1$ as in Proposition 4. For any direct B-right decomposition

$$
A=\mathfrak{R}_{1}+\mathfrak{R}_{2}+\cdots+\mathfrak{R}_{n}
$$

of $A$, we obtain a direct B-left decomposition

$$
A=\mathfrak{Q}_{1}+\mathfrak{Q}_{2}+\cdots+\mathfrak{Q}_{n}
$$

with $\mathfrak{Q}_{\nu}=L_{\pi} \Re_{\nu}^{\prime}\left(\mathfrak{R}_{\nu}^{\prime}=\Re_{1}+\cdots+\Re_{\nu-1}+\Re_{\nu+1}+\cdots+\Re_{n}\right)$. We have, for $\nu=1$, ..., $n$,

$$
\Re_{\nu}=R_{\pi} L_{\pi} \Re_{\nu}
$$

Further, $\mathfrak{L}_{\nu} \approx \operatorname{Hom}_{B, \beta}\left(\mathfrak{R}_{\nu}, B_{B}\right)$ (B-left), $\mathfrak{R}_{\nu} \approx \operatorname{Hom}_{B, \beta}{ }^{-1}\left(\mathfrak{L}_{\nu},{ }_{B} B\right)$ (B-right) if $A$ is $\beta$-Frobenius over $B$ and $\pi$ is $(B, 1)-(B, \beta)$-bilinear.

Proof. With $\beta$ as prescribed in the last part of the proposition we see, 
from (11), that $\operatorname{Hom}_{B, \beta}\left(A_{B}, B_{B}\right)$ is naturally ( $B$-left) isomorphic to the direct sum

$$
\operatorname{Hom}_{R, \beta}\left(\Re_{1}, B_{\beta}\right)+\cdots+\operatorname{Hom}_{R, \beta}\left(\Re_{n}, B_{B}\right) .
$$

Here $\operatorname{Hom}_{B, \beta}\left(\Re_{\nu}, B_{B}\right)$ corresponds to the submodule of $\operatorname{Hom}_{B, \beta}\left(A_{i}, B_{B}\right)$ consisting of all elements of $\operatorname{Hom}_{B, \beta}\left(A_{\beta}, B_{B}\right)$ mapping $\mathfrak{R}_{2}^{\prime}$ into 0 . Turning from $\operatorname{Hom}_{B, \beta}\left(A_{B}, B_{B}\right)$ to $A$ by $\Phi^{-1}$ we obtain a direct $B$-left decomposition (12) where $\mathscr{Q}_{2}$ consists of all elements $a$ of $A$ such that $(\mathscr{D} a) \mathfrak{R}_{2}^{\prime}=0$. The last relation means (D1) $a \Re_{2}^{\prime}=0$, i.e. $a \in \mathrm{L}_{\pi} \mathfrak{R}_{2}^{\prime}$.

By symmetry we get a $B$-right decomposition

$$
A=R_{\pi} \mathfrak{Q}_{1}^{\prime}+\cdots+R_{\pi} \mathfrak{Q}_{n}^{\prime}
$$

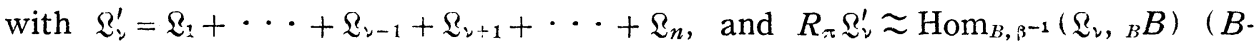
right). For $\mu \neq \nu$ we have $\mathfrak{R}_{\mu}^{\prime} \supseteqq \Re_{\nu}$ whence $\pi \mathbb{R}_{\mu} \Re_{\nu}=0$. Hence $\pi \mathbb{R}^{\prime} \Re_{\nu}=0$ whence $\Re_{\nu} \subseteq R_{\pi} \Omega_{!}^{\prime}$. From this and the direct decompositions (11), (14) we have

$$
\mathfrak{R}_{2}=R_{\pi} \mathfrak{S}_{\nu}^{\prime} .
$$

Hence $R_{\pi} L_{\pi} R_{\nu}=R_{\pi} L_{\pi} R_{\pi} \mathcal{R}_{\prime \prime}^{\prime}$. The right-hand side is equal to $R_{\pi} L_{\nu}^{\prime}$, by a general property of so-called Galois correspondences. So we get (13), again by (15).

Example 1. Let $B$ be a ring which is a direct sum of mutually orthogonal subfields $B_{1}, B_{2}$. Let $A$ be the ring of all vectors $x=\left(b_{1}, b, b^{\prime}\right)$ with $b_{1} \in B_{1}$ and $b, b^{\prime} \in B_{2}$, the addition and multiplication in $A$ being defined componentwise. Regard the set of $x$ with $b=b^{\prime}$ as $B$. It is easy to see that $A$ is a 1 . Frobenius extension of $B$; set e.g. $\pi x=b_{1}+b+b^{\prime}$. But $A$ is not a Frobenius extension of $B$ in the old sense (as in [10] and [13]), since $A$ is not $B$-right (say) free.

In this example, however, $A$ is a direct sum of two mutually orthogonal rings (consisting respectively of vectors of form $\left(b_{1}, 0,0\right)$ or $\left.\left(0, b, b^{\prime}\right)\right)$ which are Frobenius in the old sense over $B_{1}, B_{2}$ respectively.

Example 2. Let $A$ be the ring of all matrices of form

$$
x=\left(\begin{array}{lll}
\left(\begin{array}{ll}
\xi & 0 \\
\zeta & \eta
\end{array}\right) & \\
& & \\
& & \left(\begin{array}{ll}
\eta & 0 \\
\omega & \xi
\end{array}\right)
\end{array}\right)
$$

in a field $K$; for simplicity's sake we write $x=[\xi, \eta, \zeta, \omega]$. Let $B$ be the subring of $A$ consisting of all $x$ with $\zeta=\omega=0 . e_{1}=[1,0,0,0], e_{2}=[0,1,0,0]$ are 
two primitive idempotents in $B$. $B$ has an automorphism $\beta$ which interchanges $\xi$ with $\eta$ (and hence $e_{1}$ with $e_{2}$ ). Now, set

$$
\pi x=\omega e_{1}+\zeta e_{2} .
$$

If $\tilde{x}=[\tilde{\xi}, \tilde{\eta}, \widetilde{\zeta}, \widetilde{\omega}]$ then $x \widetilde{x}=[\xi \tilde{\xi}, \eta \widetilde{\eta}, \zeta \widetilde{\xi}+\eta \widetilde{\zeta}, \omega \widetilde{\eta}+\xi \widetilde{\omega}]$ and hence

$$
\pi x \widetilde{x}=(\omega \tilde{\eta}+\xi \widetilde{\omega}) e_{1}+(\zeta \widetilde{\xi}+\eta \widetilde{\zeta}) e_{2} .
$$

From this relation we see easily that $\pi$ is a $(B, 1)-(B, \beta)$-homomorphism of ${ }_{B} A_{B}$ to $B$. From the same relation we also see readily that the condition $i_{r}$ ) in Proposition 4 holds. Hence we have a $\left(B-A^{-}\right)$monomorphism $a \rightarrow \varphi_{a}=\pi^{a}$ of ${ }_{B} A_{A}$ into $\operatorname{Hom}_{B, \beta}\left(A_{B}, B_{B}\right)$. On the other hand, 1 and $u=[0,0,1,1]$ form a free $B$-right basis of $A$ (and, hence, $\left.1_{r}\right)$ ) is satisfied. Therefore, $\operatorname{Hom}_{B, \beta}\left(A_{B}, B_{B}\right)$ is a free $B$-left-module of rank 2 . Since $(1, u)$ is also a free $B$-left basis of $A$, the map $a \rightarrow \varphi_{a}=\pi^{a}$ is epimorphic too. $A$ is thus a 2. Frobenius extension of $B$ with respect to $\beta$. (This we may verify more rapidly by considering $B$-right and $B$-left bases $(1, u)$ and $(u, 1)$, dual to each other in the sense of Propositions 10,12 below).

But $A$ is not 1. Frobenius over $B$, because our automorphism $\beta$ of $B$ can not be induced by a transformation by a regular element in $A$ as we readily see.

\section{§ 3. Dimensions in Frobenius extensions}

With an extension $A$ of $B$, an automorphism $\beta$ of $B$, and with a $B$-leftmodule $\mathrm{m}$, we define an $A$-left-homomorphism

$$
\tau: A \otimes_{B} \mathrm{~m} \rightarrow \operatorname{Hom}_{B, \beta-1}\left(\operatorname{Hom}_{B, \beta}\left(A_{B}, B_{B}\right), \mathrm{m}\right)
$$

by

$$
(\tau(a \otimes u))=\left(\beta^{-1} \varphi a\right) u
$$

$\left(a \in A, u \in \mathrm{m}, \varphi \in \operatorname{Hom}_{B, \beta}\left(A_{B}, B_{B}\right)\right)$; the verification is similar as in (3), (4). If $1_{r}$ ) is the case, this homomorphism $\tau$ is an isomorphism. If $A$ is $\beta$-Frobenius over $B$, then we have thus

$$
\begin{aligned}
A \otimes_{B} \mathrm{~m} & \approx \operatorname{Hom}_{B, \beta-1}\left(\operatorname{Hom}_{B, \beta}\left(A_{B}, B_{B}\right), \mathfrak{m}\right) \\
& \approx \operatorname{Hom}_{B, \beta-1}\left({ }_{B} A, \mathrm{~m}\right) \quad(A \text {-left }) .
\end{aligned}
$$

(This is a generalization of the relation (6) (which was made explicit in (7)), but the generalization becomes complete only when we consider a $B$ - $B$-module $m$ and we consider $A-B$ - isomorphisms instead of mere $A$-left-isomorphisms). 
We combine our isomorphism

$$
A \otimes_{B} \mathrm{~m} \approx \operatorname{Hom}_{B, \beta^{-1}}\left({ }_{B} A, \mathrm{~m}\right) \quad(A \text {-left })
$$

first with [1], VI, Prop. 4.14 to obtain (cf. [4], [6])

Lemma 7. With a $\beta$-Frobenius extension $A$ of $B$ we have

$$
\operatorname{Ext}_{A}^{q}\left(\mathfrak{L}, A \otimes_{B} \mathfrak{m}\right) \approx \operatorname{Ext}_{B}^{q}\left(\mathfrak{L},\left(\beta^{-1}, \mathfrak{m}\right)\right)
$$

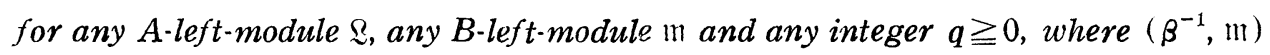
denotes the B-left- module whose structure is obtained from the B-left-module structure of $m$ with elements of $B$ acted by $\beta^{-1}$ before operating.

Proof. Thus

$$
\begin{aligned}
& \operatorname{Hom}_{A}\left(\mathbb{L}, A \otimes_{B} \mathrm{~m}\right) \approx \operatorname{Hom}_{A}\left(\mathbb{L}, \operatorname{Hom}_{B, \beta^{-1}}\left({ }_{B} A, \mathrm{~m}\right)\right) \\
& \quad \approx \operatorname{Hom}_{B, \beta^{-1}}\left(A \otimes_{A} \mathfrak{L}, \mathrm{m}\right)=\operatorname{Hom}_{B, \beta^{-1}}(\mathbb{L}, \mathrm{m}) \\
& \approx \operatorname{Hom}_{B}\left(\mathfrak{L},\left(\beta^{-1}, \mathrm{~m}\right)\right),
\end{aligned}
$$

which proves (19) for $q=0$. On replacing here $\mathfrak{Z}$ with an $A$-(left-) projective resolution $\mathfrak{X}$ of $\mathfrak{Z}$ and observing that $\mathfrak{X}$ is also a $B$-projective resolution of $\mathfrak{L}$, we obtain, in passing to homology, (19) for $q>0$.

Theorem 8. Let $A$ be a 2. Frobenius extension of $B$. Then

$$
\text { 1. } \operatorname{dim}_{A} \mathfrak{Q}=1 . \operatorname{dim}_{B} \mathfrak{Q}
$$

for any A-left-module $\mathfrak{Q}$ with $1 . \operatorname{dim}_{A} \mathfrak{Q}<\infty$.

Proof runs again similarly as in Eilenberg-Nakayama [4] or Hirata [6]. Thus, set $1 \cdot \operatorname{dim}_{A} \mathfrak{Q}=n<\infty$. There exists then an $A$-left-module $\mathfrak{R}$ with $\operatorname{Ext}_{A}^{n}(\mathfrak{L}, \mathfrak{R}) \neq 0$. Consider an exact sequence

$$
0 \rightarrow \mathfrak{N} \rightarrow \mathfrak{F} \rightarrow \mathfrak{M} \rightarrow 0
$$

with $\widetilde{F} A$-left-free. Since $\operatorname{Ext}_{A}^{n+1}(\mathfrak{R}, \mathfrak{R})=0$ it follows that the induced homomorphism $\operatorname{Ext}_{A}^{n}(\mathfrak{L}, \mathfrak{F}) \rightarrow \operatorname{Ext}_{A}^{n}(\mathfrak{L}, \mathfrak{R})$ is an epimorphism, and therefore $\operatorname{Ext}_{A}^{n}(\mathfrak{L}, \mathfrak{F})$ $\neq 0$. Now, $\widetilde{F} \approx A \otimes_{B}$ in with a $B$-left-free module $\mathrm{m}$, and, by our lemma, $\operatorname{Ext}_{B}^{n}\left(\mathscr{L},\left(\beta^{-1}, \mathrm{~m}\right)\right) \approx \operatorname{Ext}_{A}^{n}\left(\mathfrak{L},\left(A \otimes_{B} \mathrm{~m}\right)\right)=\operatorname{Ext}_{A}^{n}(\mathfrak{L}, \mathfrak{F})$. Hence $\operatorname{Ext}_{B}^{n}\left(\mathfrak{L},\left(\beta^{-1}, \mathfrak{m}\right)\right)$ $\neq 0$ which proves $1 \cdot \operatorname{dim}_{B} \mathfrak{Q} \geqq n=1 \cdot \operatorname{dim}_{A} \mathfrak{Q}$. That $1 \cdot \operatorname{dim}_{B} \mathfrak{Q} \leqq 1 \cdot \operatorname{dim}_{A} \mathfrak{Q}$ is clear since $A$ is $B$-left-projective.

Theorem 9. Let $A$ be a 2. Frobenius extension of $B$. Then 


$$
\text { 1. inj. } \begin{aligned}
\operatorname{dim}_{B} m & \geqq 1 \text { inj. } \operatorname{dim}_{A}\left(A \otimes_{B} \mathrm{~m}\right) \\
& \geqq 1 \text {. inj. } \operatorname{dim}_{B}\left(A \otimes_{B} \mathrm{~m}\right) .
\end{aligned}
$$

for any $B$-left-module $\mathrm{m}$. If further the $B$-B-module $B$ is $\gamma$-1-isomorphic to a direct summand of the $B$-B-module $A, \gamma$ being some automorphism of $B$, then the inequalities in (22) become equalities.

Proof. The first inequality in (22) follows from Lemma 7. As for the second one, we have generally 1. inj. $\operatorname{dim}_{A} \mathfrak{l} \geqq 1$.inj. $\operatorname{dim}_{B} \mathfrak{L}$ for any $A$-left-module $\mathfrak{L}$, since every $A$-injective resolution of $\mathfrak{Z}$ is also a $B$-injective resolution because of $\left.1_{l}\right){ }^{4}$ ) The last part of the theorem is clear, since under the assumption the $B$-left-module $A \otimes_{B} \mathrm{~lm}$ has a direct $B$-left-summand $\gamma^{-1}$-isomorphic to "m.

Setting $\mathrm{m}=B$ we have

Corollary 10. Let $A$ be a 2. Frobenius extension of $B$. Then

$$
\text { 1. inj. } \operatorname{dim}_{B} B \geqq 1 \text { inj. } \operatorname{dim}_{A} A \geqq 1 \text { inj. } \operatorname{dim}_{B} A \text {. }
$$

Under the same additional condition as in the last part of Theorem 9 we have equalities in (23).

Corollary 11. Let $A$ be 2. Frobenius over $B$. If $B$ is left self-injective, then $A$ is so too. In particular, if $B$ is a quasi-Frobenius ring (in the sense of [12]), then $A$ is a such too. Under the additional condition as in the last part of Theorem 9 the converses of these contentions are also true.

For the characterization of quasi-Frobenius rings by (left) self-injectivity cf. [8], [4].

Now, combining the isomorphism (18) with [1], VI, Prop. 4.13, we have (cf. [5])

Lemma 7'. With a $\beta$-Frobenius extension $A$ of $B$ we have

$$
\operatorname{Ext}_{A}^{q}\left(\operatorname{Hom}_{B}\left({ }_{B} A, \mathfrak{m}\right), \mathfrak{l}\right) \approx \operatorname{Ext}_{B}^{q}\left(\left(\beta^{-1}, \mathfrak{m}\right), \mathfrak{l}\right)
$$

for any A-left-module $\&$, any B-left-module $\mathrm{m}$ and any integer $q \geqq 0$.

Proof is, thus, quite similar to that of Lemma 7. We have, by (18),

$$
\operatorname{Hom}_{A}\left(\operatorname{Hom}_{B, \beta-1}\left({ }_{B} A, \text { in }\right), \mathfrak{\&}\right) \approx \operatorname{Hom}_{A}\left(A \otimes_{B} \mathrm{~m}, \mathfrak{\&}\right)
$$

4) The same follows also from 1 ), or even from a weaker condition that $A$ is $B$-right. flat; see [1], VI, Ex. 10. 


$$
\approx \operatorname{Hom}_{B}\left(\mathfrak{m}, \operatorname{Hom}_{A}(A, \mathfrak{l})\right)=\operatorname{Hom}_{B}(\mathfrak{m}, \mathfrak{\Omega}) .
$$

Replacing $m$ with $\left(\beta^{-1}, \mathfrak{m}\right)$ we obtain the case $q=0$ of (24). Replacing then $\mathbb{Z}$ with its $A$-injective resolution $\mathfrak{Y}$, observing that $\mathfrak{Y}$ is also a $B$-injective resolution, because of $\left.1_{l}\right)$, ${ }^{5)}$ and passing to homology, we get (23) for $q>0$.

Dually to Theorem 8 we have

Theorem $8^{\prime}$. Let $A$ be 2. Frobenius over $B$. Then

$$
\text { 1.inj. } \operatorname{dim}_{A} \mathfrak{Q}=1 \text {. inj. } \operatorname{dim}_{B} \mathfrak{Z}
$$

for any A-left-module $\&$ with 1. inj. $\operatorname{dim}_{A} \mathfrak{Q}<\infty$.

Proof. We set l.inj. $\operatorname{dim}_{A} \mathfrak{Q}=n<\infty$, and take an $A$-left-module $\mathfrak{M}$ with $\operatorname{Ext}_{A}^{n}(\mathfrak{M}, \mathfrak{L}) \neq 0$. Let $\mathrm{m}$ be an injective $B$-left-module having a $B$-leftsubmodule isomorphic to the $B$-left-module $\mathfrak{M}$. Then $\operatorname{Hom}_{B}\left({ }_{B} A, \mathfrak{m}\right)$ is, by [1], VI, Prop. 1.4 (cf. [2] for a different proof), an injective $A$-left-module and contains a $A$-left-submodule isomorphic to $\operatorname{Hom}_{B}\left({ }_{B} A, \mathfrak{M}\right)$. The last module has in turn an $A$-left-submodule $\operatorname{Hom}_{A}\left({ }_{A} A, \mathfrak{M}\right) \approx \mathfrak{M}$. Thus we have proved the existence of exact sequence

$$
0 \rightarrow \mathfrak{M} \rightarrow \operatorname{Hom}_{B}\left({ }_{B} A, \mathrm{~m}\right) \rightarrow \mathfrak{R} \rightarrow 0
$$

of $A$-left-modules, where $\operatorname{Hom}_{B}\left({ }_{B} A, \mathfrak{m}\right)$ is $A$-left-injective. On considering the exact sequence $\operatorname{Ext}_{A}^{n}\left(\operatorname{Hom}_{B}\left({ }_{B} A, \mathfrak{m}\right), \mathfrak{I}\right) \rightarrow \operatorname{Ext}_{A}^{n}(\mathfrak{M}, \mathfrak{L}) \rightarrow \operatorname{Ext}_{A}^{n+1}(\mathfrak{N}, \mathfrak{L})=0$ and on applying (24) we can, now prove $\operatorname{Ext}_{B}^{n}\left(\left(\beta^{-1}, \mathfrak{m}\right), \mathfrak{L}\right) \neq 0$. Hence 1. inj. ${ }_{B} \mathfrak{L} \geqq n$

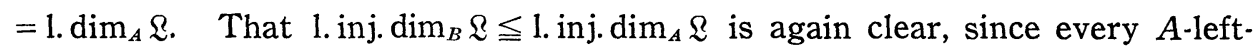
injective resolution is by $1_{l}$ ) also a $B$-left-injective resolution. ${ }^{6)}$

\section{Theorem 9'. Let $A$ be 2. Frobenius over $B$. Then}

$$
\text { 1. } \begin{aligned}
\operatorname{dim}_{B} \mathfrak{m} & \geqq 1 \operatorname{dim}_{A} \operatorname{Hom}_{B}\left({ }_{B} A, \mathfrak{m}\right) \\
& \geqq 1 . \operatorname{dim}_{B} \operatorname{Hom}_{B}\left({ }_{B} A, \mathfrak{m}\right)
\end{aligned}
$$

for any B-left-module $\mathrm{m}$. If the additional condition in the last part of Theorem 9 holds, then we have equalities in (27).

Proof. The first inequality in (27) follows from Lemma $7^{\prime}$. The second one is clear from $1_{l}$ ); cf. the final part of our proof to Theorern 8 . The last con-

5) Cf. the preceding foot-note 4 ).

6) See the foot-note 4 ). 
tention in our theorem is also evident, since under the condition the $B$-leftmodule $\operatorname{Hom}_{B}\left({ }_{B} A, \mathrm{~m}\right)$ has a direct summand $\gamma^{-1}$-isomorphic to $\mathrm{m}$.

Further, we have

Lemma $7 "$. With a $\beta$-Frobenius extension $A$ of $B$ we have

$$
\operatorname{Tor}_{q}^{A}\left(\Re, \operatorname{Hom}_{B}\left({ }_{B} A, \mathfrak{m}\right)\right) \approx \operatorname{Tor}_{q}^{B}\left(\Re,\left(\beta^{-1}, \mathfrak{m}\right)\right)
$$

for any A-right-module $\mathfrak{R}$, any B-left-module $m$ and any integer $q \geqq 0$.

Proof is similar to those of Lemmas 7, $7^{\prime}$ and is thus simply to combine (18) with [1], VI, Prop. 4.1.2; replace $\Gamma, A, A, C$ there by $A, B . \Re,\left(\beta^{-1}, \mathrm{~m}\right)$.

Theorem $8^{\prime \prime}$. Let $A$ be 2. Frobenius over $B$. Then

$$
\text { 1. w. } \operatorname{dim}_{A} \mathfrak{Q}=1 \text {. w. } \operatorname{dim}_{B} \mathfrak{Q}
$$

for any A-left-module $\mathfrak{Q}$ with 1.w. $\operatorname{dim}_{A} \mathfrak{Q}<\infty$.

Proof. It is sufficient to prove a similar equality for an $A$-right-module $\Re$ with r.w. $\operatorname{dim}_{A} \mathfrak{R}<\infty$. Denoting the last dimension by $n$, let $\mathfrak{M}$ be an $A$-leftmodule with $\operatorname{Tor}_{n}^{A}(\mathfrak{M}, \mathfrak{M}) \neq 0$. Let $m$ be as in the proof to Theorem $8^{\prime}$. We have the exact sequence (26) with $\operatorname{How}_{B}\left({ }_{B} A, \mathrm{~m}\right) A$-left-injective. The corresponding homology exact sequence $\operatorname{Tor}_{n+1}^{A}(\mathfrak{X}, \mathfrak{R})(=0) \rightarrow \operatorname{Tor}_{n}^{A}(\mathfrak{R}, \mathfrak{R}) \rightarrow \operatorname{Tor}_{n}^{A}(\mathfrak{R}$, $\left.\operatorname{Hom}_{B}\left({ }_{B} A, m\right)\right)$ implies that its last term does not vanish. But, this last term is by Lemma $7^{\prime \prime}$ isomorphic to $\operatorname{Tor}_{n}^{B}\left(\Re,\left(\beta^{-1}, \mathfrak{m}\right)\right)$. Hence r.w. $\operatorname{dim}_{B} \mathfrak{R} \geqq n$. That conversely r.w. $\operatorname{dim}_{B} \Re \leqq$ r.w. $\operatorname{dim}_{A} \Re$ follows from $1_{r}$ ) (or even from the $B$-right-flatness ; cf. [1], VI, Ex. 10).

Theorem $9^{\prime \prime}$. Let $A$ be 2. Frobenius over B. Then

$$
\text { 1.w. } \begin{aligned}
\operatorname{dim}_{B} \mathrm{~m} & \geqq 1 \text { w. } \operatorname{dim}_{A} \operatorname{Hom}_{B}\left({ }_{B} A, \mathfrak{m}\right) \\
& \geqq 1 \text { w. } \operatorname{dim}_{B} \operatorname{Hom}_{B}\left({ }_{B} A, \mathfrak{m}\right) .
\end{aligned}
$$

If the additional condition in the last part of Theorem 9 holds, then we have equalities in (30).

Proof. (30) follows from Lemma 7" and a general inequality whose (leftright) counter-part was used already in the final part of the above proof to Theorem $8^{\prime \prime}$.

Remark. The inequalities which we obtain by exchanging $A \otimes_{B} \mathrm{~m}, \mathrm{Hom}_{B}$ $\left({ }_{3} A, \mathrm{~m}\right)$ in $(22),(27),(30)$ are rather trivial ones holding under the conditions 
$\left.1_{l}\right), 1_{r}$ ) only, ${ }^{i}$ contrary to the deeper lying first inequalities in (23), (27), (30).

\section{§. Quasi-free Frobenius extensions}

A left-(resp. right-) module over a ring $B$ is called quasi-free, when it is a direct sum of $B$-left-(right-) submodules each of which is $B$-isomorphic to a left (right) ideal of $B$ generated by an idempotent in $B$; $\operatorname{cf}[3]$. We remark that if $e$ is an idempotent in $B$ and $\beta$ is an automorphism of $B$, then

$$
\begin{array}{ll}
\operatorname{Hom}_{B, \beta}\left((e B)_{B}, B_{B}\right) \approx B \beta e(=\beta B e)^{81} & (B \text {-left }), \\
\operatorname{Hom}_{B, \beta}\left({ }_{B}(B e),{ }_{B} B\right) \approx(\beta e) B(=\beta e B) & (B \text {-right }),
\end{array}
$$

as we easily see in customary way.

Now, we strengthen $1_{r}$ ), $1_{l}$ ) (in Proposition 1) into the conditions :

$\left.1_{r}^{\circ}\right)$ the $B$-right-module $A$ is finitely generated and quasi-free,

$1_{l}$ ) the $B$-left-module $A$ is finitely generated and quasi-free,

and obtain

Proposition $1^{\circ}$. For a ring $A$ and its subring $B$ the set of conditions $1_{r}^{\circ}$ ), $2_{r}$ ) is equivalent to the set $\left.1_{i}\right), 2_{r}$ ).

Proof runs similarly as in that of Proposition 1 if we observe (31), (32).

Definition. A ring $A$ is said to be a quasi-free 2. Frobenius extension of its subring $B$ when the equivalent sets of conditions $\left.1_{r}^{\circ}\right), 2_{r}$ ) and $\left.1_{l}^{i}\right), 2_{l}$ ) are fulfilled. Termilnologies as "quasi-free $\beta$-Frobenius", "quasi-free 1. Frobenius" are defined similarly.

Proposition 10. Let $A$ be a quasi-free $\beta$-Frobenius extension of $B$, and let

$$
A=R_{1}+\cdots+R_{n} \quad\left(R_{\imath}=v_{\nu} B\right)
$$

be a direct decomposition of $A$ into $B$-right modules such that each $R_{\nu}=v_{\nu} B$ $(\nu=1, \ldots, n)$ is $B$-right-isomorphic to a right-ideal $e_{\nu} B$ of $B$ generated by an idempotent $e_{\nu}$ in $B$ by the correspondence $v_{\nu} y \rightarrow e_{\nu} y(y \in B)$. Then, for any given $B$-A-isomorphism $\Phi$ of ${ }_{B} A_{A}$ and $\operatorname{Hom}_{B, \beta}\left(A_{B}, B_{B}\right)$ there exists a system of elements $u_{1}, \ldots, u_{n}$ in $A$ (dual to $v_{1}, \ldots, v_{n}$ ) such that

7) Some even under a weaker condition of $B$-flatness of $A$. Cf. [1], VI, Ex. 10; it may not be useless to remark that (p) $A$ in the last formula there (1st. edition) should read (p) A.

8) Here, and for the followings, we recall our agreement made in foot-note 2 ). 


$$
\left(\Phi u_{\mu}\right) v_{\nu}=\delta_{\mu \nu} \beta e_{\mu}
$$

We have a direct decomposition

$$
A=B u_{1}+\cdots+B u_{n}
$$

and each component $B u_{\nu}$ is B-left-isomorphic to $B \beta e_{\nu}$ by the correspondence $y u_{\nu}$ $\rightarrow y \beta e_{\nu}(y \in B)$.

Proof. Denote by $\varphi_{\nu}$ the $B$-right-homomorphism of $A$ into $B$ such that $\varphi_{\nu} v_{\nu}=e_{\nu}$ and $\varphi_{\nu} v_{\mu}=0$ for $\mu \neq \nu$. Observing that every $B$-right-homomorphic image in $B$ of $v_{\nu} B$ (whence of $e_{\nu} B$ ) is obtained from $e_{\nu} B$ by a left-multiplication of an element of $B$, we obtain then readily a direct decomposition

$$
\operatorname{Hom}_{B, \beta}\left(A_{B}, B_{B}\right)=\beta B \varphi_{1}+\cdots+\beta B \varphi_{n}=B \beta \varphi_{1}+\cdots+B \beta \varphi_{n} .
$$

We have $e_{\nu} \varphi_{\nu}=\varphi_{\nu}, B \varphi_{\nu} \approx B e_{\nu}(B$-left $)$, and $\beta B \varphi_{\nu} \approx \beta B e_{\nu}\left(=B \beta e_{\nu}\right)(B(=\beta B)$-left $)$. Observing $\beta \varphi_{\nu} \in \operatorname{Hom}_{B, \beta}\left(A_{B}, B_{B}\right)$, set

$$
u_{\nu}=\emptyset^{-1} \beta \varphi_{\nu} \quad(\in A) .
$$

Then we obtain our assertions readily (cf. Proof to Proposition 6). In particular, we have $\left(\Phi u_{\mu}\right) v_{\nu}=\left(\beta \varphi_{\mu}\right) v_{\nu}=\beta \varphi_{\mu} v_{\nu}=\delta_{\mu \nu} \beta e_{\mu}$.

Remark. With the $(B, 1)-(B, \beta)$-homomorphism of $A$ into $B \pi=\Phi 1$ as in Proposition 4 the relation (34) may be expressed as

$$
\pi u_{\mu} v_{\nu}=\delta_{\mu \nu} \beta e_{\mu}
$$

observe $\Phi u_{\mu}=\Phi 1 u_{\mu}=(\Phi 1)^{u_{\mu}}=\pi^{u_{\mu}}$ as operators on $A$. In terms of the scalar product \langle\rangle defined by $\langle x, y\rangle=\pi x y$ as in our observation following Corollary 5 , our relation is evidently expressed also as

$$
\left\langle u_{\mu}, v_{\mu}\right\rangle=\delta_{\mu \nu} \beta e_{\mu} .
$$

We have also

$$
1=\sum_{\nu} v_{\nu} \beta^{-1} \pi u_{\nu}=\sum_{\nu}\left(\pi v_{\nu}\right) u_{\nu}
$$

For, if we put $1=\sum_{\nu} v_{\nu} y_{\nu} \quad\left(y_{\nu} \in e_{\nu} B\right)$, we have $\pi u_{\mu}=\pi u_{\mu} \sum_{\nu} v_{\nu} y_{\nu}=\sum_{\nu}\left(\pi u_{\mu} v_{\nu}\right) \beta y_{\nu}$ $=\left(\beta e_{\mu}\right) \beta y_{\nu}=\beta e_{\mu} y_{\nu}=\beta y_{\nu}$, which proves the first half of $(40)$. The second half of $(40)$ is proved similarly; we remark that $\beta$ does not appear explicitly in the last sum in $(40)$.

Proposition 11. Let $v_{\nu}, u_{\nu}(\nu=1, \ldots, n)$ be as in Proposition 10. For 
every element $x$ of $A$ each product $x v_{\nu}$ is expressed uniquely as

$$
x v_{\nu}=\sum_{\mu=1}^{n} v_{\mu} y_{\mu \nu} \quad\left(y_{\mu \nu} \in e_{\mu} B e_{\nu}\right)
$$

and we have

$$
u_{\mu} x=\sum_{\nu=1}^{n}\left(\beta y_{\mu \nu}\right) u_{\nu}
$$

Proof. The first half is rather evident. We have then

$$
\text { (Ф1) } u_{\mu} x v_{\nu}=(\Phi 1) u_{\mu} \sum_{\kappa=1}^{n} v_{\kappa} y_{\kappa \nu}=\sum_{\kappa} \delta_{\mu \kappa} \beta y_{\kappa \nu}=\beta y_{\kappa \nu},
$$

i.e. $\pi u_{\mu} x v_{\nu}=\beta y_{\kappa \nu}$. Similarly we obtain $\pi u_{\mu} x v_{\nu}=z_{\mu \nu}$ if we set $u_{\mu} x=\sum_{\nu=1}^{n} z_{\mu \nu} u_{\nu}$ $\left(z_{\mu \nu} \in\left(\beta e_{\mu}\right) B \beta e_{\nu}\right)$. We must have $z_{\mu \nu}=\beta y_{\mu \nu}$, and hence (42).

Conversely we have

Proposition 12. Let $B$ be a subring of a ring $A$. Let there be a system $v_{\nu}$, $u_{\nu}(\nu=1, \ldots, n)$ of elements in $A$ such that the direct decompositions (33), $(35)$, i.e.

$$
\begin{aligned}
A & =v_{1} B+\cdots+v_{n} B \\
& =B u_{1}+\cdots+B u_{n}
\end{aligned}
$$

hold, that we have isomorphisms $v_{\nu} B \approx e_{\nu} B$ (B-right), $B u_{\nu} \approx B \beta e_{\nu}$ (B-left) by $v_{\nu} \leftrightarrow e_{\nu}, u_{\nu} \leftrightarrow \beta e_{\nu}$, with idempotents $e_{\nu}$ in $B$ and, that (41) (with $x \in A$ ) entails (42). Then $A$ is $\beta$-Frobenius over $B$.

Proof. The condition $1_{r}$ ) of Proposition 1 is evident by (33) and $v_{\nu} B \approx e_{\nu} B$. To verify $2_{r}$ ) we observe that the same decomposition (33) and isomorphism $v_{\nu} B \approx e_{\nu} B\left(v_{\nu} \leftrightarrow e_{\nu}\right)$ imply the existence of $n \beta$-homomorphisms $\varphi_{\nu}$ of $A_{B}$ into $B$ such that

$$
\varphi_{\nu} v_{\mu}=\delta_{\nu \mu} \beta e_{\nu}
$$

Associating each $u_{\nu}$ with $\varphi_{\nu}$ and observing (35) and $B u_{\nu} \approx B \beta e_{\nu}\left(u_{\nu} \leftrightarrow \beta e_{\nu}\right)$, we readily obtain a $B$-left isomorphism of ${ }_{B} A$ and $\operatorname{Hom}_{B, \beta}\left(A_{B}, B_{B}\right)$. It is also an $A$-right isomorphism by virtue of (41), (42).

Theorem 13. Let $A$ be a $\beta$-Frobenius extension of $B$, and let $v_{\nu}, u_{\nu}(\nu=1$, $\ldots, n$ ) be as in Proposition 10 (with a given $B$-A-isomorphism $\Phi$ of ${ }_{B} A_{A}$ to $\left.\operatorname{Hom}_{B, \beta}\left(A_{B}, B_{B}\right)\right)$.

Suppose that the automorphism $\beta$ of $B$ can be extended to an automorphism 
of $A$, which we denote also by $\beta$. Then the sum

$$
w=\sum_{\nu=1}^{n}\left(\beta v_{\nu}\right) u_{\nu}
$$

in A satisfies

$$
(\beta x) w=w x \quad \text { for } \text { all } x \in A \text {. }
$$

The element $w$ is determined uniqualy by $\Phi,(B$-right) isomorphism type of the direct decomposition (33), and the extended automorphism $\beta$ of $A$, independent of the choice of $v_{\nu}, u_{\nu}$.

Proof. The formula (44) follows readily from (41), (42) of Proposition 11. Let next $v_{\nu}^{\prime}, u_{\nu}^{\prime}(\nu=1, \ldots, n)$ be a second system of elements in $A$ such that we have direct decompositions

$$
\begin{aligned}
A & =v_{1}^{\prime} B+\cdots+v_{n}^{\prime} B \\
& =B u_{1}^{\prime}+\cdots+B u_{n}^{\prime}
\end{aligned}
$$

wiun $v_{\nu}^{\prime} B$ (resp. $\left.B u_{\nu}^{\prime}\right) B$-right (resp. left) isomorphic to $e_{\nu}^{\prime} B$ (resp. $B \beta e_{\nu}^{\prime}$ ) by $v_{\nu}^{\prime}$ $\rightarrow e_{\nu}^{\prime}$ (resp. $\left.u_{\nu}^{\prime} \rightarrow \beta e_{\nu}^{\prime}\right), e_{\nu}^{\prime}$ idempotent, and $\left(\Phi u_{\mu}^{\prime}\right) v_{\nu}^{\prime}=\delta_{\mu \nu} \beta e_{\mu}^{\prime}$. We first assume that $v_{\nu} B=v_{\nu}^{\prime} B, B u_{\nu}=B u_{\nu}^{\prime}$ hold for every $\nu=1, \ldots, n$. Then we have $v_{\nu}^{\prime}=v_{\nu} z_{\nu}$, $u_{\nu}^{\prime}=y_{\nu} u_{\nu}$ with $z_{\nu} \in e_{\nu} B e_{\nu}^{\prime}, y_{\nu} \in\left(\beta e_{\nu}^{\prime}\right) B \beta e_{\nu}$. Then

$$
y_{\nu} \beta z_{\nu}=y_{\nu}\left(\beta e_{\nu}\right) \beta z_{\nu}=y_{\nu}\left(\pi u_{\nu} v_{\nu}\right) \beta z_{\nu}=\pi y_{\nu} u_{\nu} v_{\nu} z_{\nu}=\pi u_{\nu}^{\prime} v_{\nu}^{\prime}=\beta e_{\nu}^{\prime} .
$$

Moreover, we have also $u_{\nu}=w_{\nu} u_{\nu}^{\prime}$ with $w_{\nu} \in\left(\beta e_{\nu}\right) B \beta e_{\nu}^{\prime}$, and from $u_{\nu}=w_{\nu} y_{\nu} u_{\nu}$, $u_{\nu}^{\prime}=y_{\nu} w_{\nu} u_{\nu}^{\prime}$ we obtain readily $w_{\nu} y_{\nu}=\beta e_{\nu}, y_{\nu} w_{\nu}=\beta e_{\nu}^{\prime}$. Hence

$$
\beta z_{\nu}=\left(\beta e_{\nu}\right) \beta z_{\nu}=w_{\nu} y_{\nu} \beta z_{\nu}=w_{\nu} \beta e_{\nu}^{\prime}=w_{\nu}
$$

So $\left(\beta z_{\nu}\right) y_{\nu}=w_{\nu} y_{\nu}=\beta \boldsymbol{e}_{\nu}$ and we have

$$
\sum\left(\beta v_{\nu}^{\prime}\right) u_{\nu}^{\prime}=\sum\left(\beta v_{\nu} z_{\nu}\right) y_{\nu} u_{\nu}=\sum\left(\beta v_{\nu}\right)\left(\beta e_{\nu}\right) u_{\nu}=\sum\left(\beta v_{\nu}\right) u_{\nu} .
$$

Next we assume $e_{\nu}^{\prime}=e_{\nu}(\nu=1, \ldots, n)$ (hence $v_{\nu}^{\prime} B \approx v_{\nu} B, B u_{\nu}^{\prime} \approx B u_{\nu}$ (but not necessarily $\left.v_{\nu}^{\prime} B=v_{\nu} B, B u_{\nu}^{\prime}=B u_{\nu}\right)$ ). Put $v_{\nu}^{\prime}=\sum_{\mu=1}^{n} v_{\mu} z_{\mu \nu}, u_{\nu}^{\prime}=\sum_{\mu=1}^{n} y_{\nu \mu} u_{\mu}$ with $z_{\mu \nu} \in e_{\mu} B e_{\nu}, y_{\nu \mu} \in\left(\beta e_{\nu}\right) B \beta e_{\mu}$. Then $u_{\mu}^{\prime} v_{\nu}^{\prime}=\sum_{\kappa, \omega=1}^{n} y_{\mu \kappa} u_{\kappa} v_{\omega} z_{\omega \nu}$, and applying $\pi$ we have

$$
\delta_{\mu \nu} \beta e_{\mu}=\sum_{\kappa} y_{\mu \kappa} \beta z_{\kappa \nu}
$$


or

$$
\left(\begin{array}{ccc}
\beta e_{1} & & 0 \\
0 & \cdot & \beta e_{n}
\end{array}\right)=Y Z \text { with } Y=\left(y_{\mu \nu}\right), Z=\left(\beta z_{\mu \nu}\right)
$$

As we have $u_{\nu}=\sum_{\mu=1}^{n} w_{\nu \mu} u_{\mu}^{\prime}$ with some $w_{\nu \mu} \in\left(\beta e_{\nu}\right) B \beta e_{\mu}$, we have, by a habitual argument as before,

or

$$
\left(\begin{array}{ccc}
\beta e_{1} & & \\
& \cdot & 0 \\
0 & & \beta e_{n}
\end{array}\right)=Z Y
$$

Hence

$$
\delta_{\mu \nu} \beta e_{\mu}=\sum_{\kappa}\left(\beta z_{\mu \kappa}\right) y_{\kappa \nu}
$$

$$
\begin{aligned}
\sum_{\nu}\left(\beta v_{\nu}^{\prime}\right) u_{\nu}^{\prime} & =\sum_{\nu, \mu, \kappa}\left(\beta v_{\mu} z_{\mu \nu}\right) y_{\nu \kappa} u_{\kappa} \\
& =\sum_{i \nu}\left(\beta v_{\mu}\right)\left(\beta e_{\mu}\right) u_{\mu}=\sum_{\nu}\left(\beta v_{\nu}\right) u_{\nu}
\end{aligned}
$$

These considerations, combined, settle the general case where we simply assume $v_{\nu}^{\prime} B \approx v_{\nu} B$ (whence $\left.B u_{\nu}^{\prime} \approx B u_{\nu}\right)(\nu=1, \ldots, n)$.

In case $B$ is semi-primary (a ring $B$ is called semi-primary when its (Jacobson) radical $N$ is nilpotent and the residue ring $B / N$ satisfies minimum condition) every projective right (say)-module over $B$ is quasi-free; see Nagao-Nakayama [11], Eilenberg [3]. Hence every 2. Frobenius extension $A$ of $B$ is quasi-free. We have further

Corollary 14. Let $A$ be a $\beta$-Frobenius extension of a semi-primary ring $B$, where $\beta$ is an automorphism of $B$, and suppose that $\beta$ can be extended to an automorphism of $A$, denoted also by $\beta$. Then the sum $w=\sum_{\nu=1}^{n}\left(\beta v_{\nu}\right) u_{\nu}((43))$ in Theorem 13, with $v_{\nu}, u_{\nu}$ in Proposition 10, is determined uniquely by $\Phi$ and the extended automorphism $\beta$ of $A$ only, independent of the decomposition (33) and the choice of $v_{\nu}, u_{\nu}$.

Proof. Decompose each $e_{\nu}$ into a (finite) sum $e_{\nu}^{\prime}+e_{\nu}^{\prime \prime}+\cdots$ of mutually orthogonal primitive idempotents $e_{\nu}^{\prime}, e_{\nu}^{\prime \prime}, \ldots$ Put $v_{\nu}^{\prime}=v_{\nu} e_{\nu}^{\prime}, v_{\nu}^{\prime \prime}=v_{\nu} e_{\nu}^{\prime \prime}, \ldots$ and similarly $u_{\nu}^{\prime}=\beta e_{\nu}^{\prime} u_{\nu}, u_{\nu}^{\prime \prime}=\beta e_{\nu}^{\prime \prime} u_{\nu}, \ldots$ Then we have

$$
A=v_{1}^{\prime} B+v_{1}^{\prime \prime} B+\cdots+v_{n}^{\prime} B+v_{n}^{\prime \prime} B+\cdots \quad \text { (direct) }
$$

and

$$
\left(\Phi u_{\mu}^{(i)}\right) v_{\nu}^{(j)}=\delta_{\mu \nu} \delta_{i j} \beta e_{\mu}^{(i)} .
$$


Further

$$
\sum_{\nu}\left(\left(\beta v_{\nu}^{\prime}\right) u_{\nu}^{\prime}+\left(\beta v_{\nu}^{\prime \prime}\right) u_{\nu}^{\prime \prime}+\cdots\right)=\sum_{\nu}\left(\beta v_{\nu}\right) u_{\nu}
$$

This reduces the consideration of the sum $\Sigma\left(\beta v_{\nu}\right) u_{\nu}$ to a case where the idempotents $e_{2}$, are primitive. Since the direct ( $B$-right) decomposition (33) of $A$ corresponding to such a case of primitive $e_{\downarrow}$ (which is equivalent to that $B$ right modules $R_{\downarrow}$ are directly indecomposable) is unique up to ( $B$-right) isomorphisms, we have our uniqueness assertion by Theorem 13.

\section{§. Frobenius extensions over an $S$-ring}

Let $\mathbb{R}, \mathfrak{A}$ be respectively a left- and a right-module over a ring $B$. With an automorphism $\beta$ of $B$, we consider, as we did in connection of Corollary 5 in $\S 1$, a $1-\beta$-scalar product $\langle>$ of $\mathscr{L}, \mathfrak{R}$ in $B$.

If $\mathfrak{R}$ is in particular a $B$-right-module of form $\mathfrak{R}=e B$ with an idempotent $e$ in $B$, then the $B$-left-module $\operatorname{Hom}_{B, \beta}\left(\Re_{B}, B_{B}\right)$ and $\Re$ have a regular 1- $\beta$-scalar product defined by $\langle\varphi, x\rangle=\varphi x\left(x \in \Re, \varphi \in \operatorname{Hom}_{B, \beta}\left(B_{B}\right)\right) ; \operatorname{Hom}_{B, \beta}\left(\Re_{B}, B_{B}\right)$ is $B$-left isomorphic to $B \beta e=\beta B e$ and, if we identify it with this module $B \beta e$, the (1- $\beta$-) scalar product $\langle y, x\rangle$ is nothing but the product $y \beta x$ in $B$. Thus, more generally, if $\Re$ is a finitely generated quasi-free $B$-right-module, then the $B$-leftmodule

$$
\mathfrak{L}=\operatorname{Hom}_{B, \beta}\left(\Re_{B}, B_{B}\right)
$$

is quasi-free, a natural correspondence gives

$$
\Re=\operatorname{Hom}_{B, \beta^{-1}}\left({ }_{B} \mathscr{Q},{ }_{B} B\right),
$$

and, further, the pair $\mathfrak{L}, \mathfrak{R}$ has a regular $1-\beta$-scalar product. This is merely a restatement of a fact which underlay our consideration in $\S 4$. Indeed, if $\mathbb{R}, \mathfrak{R}$ are $B$-left- and $B$-right modules such that (44), (45) hold, then we obtain a regular $1-\beta$-scalar product for $\mathfrak{L}, \mathfrak{R}$ in $B$ by

$$
\langle u, v\rangle=u v
$$

on considering $u \in \mathbb{R}=\operatorname{Hom}_{B, \beta}\left(\Re_{B}, B_{B}\right)$ as a map of $\mathfrak{A}$ into $B$, and for every direct decomposition

$$
\mathfrak{R}=\sum_{i=1}^{n} \mathfrak{r}_{i}
$$

of $\Re$ inţo (a finite number of) $B$-right-submodules we obtain a direct decompo- 
sition

$$
\mathfrak{Q}=\sum_{i=1}^{n} \mathfrak{l}_{i}
$$

of $\mathfrak{Z}$ with $\mathfrak{l}_{i}$ denoting the left annihilator of $\sum_{j \neq i} \mathfrak{r}_{j}$ in $\mathfrak{Z}$ with respect to the scalar product $\langle>$ and satisfying

$$
\begin{array}{ll}
\mathfrak{l}_{i} \approx \operatorname{Hom}_{B, \beta}\left(\mathfrak{r}_{i B}, B_{B}\right) & (B \text {-left }), \\
\mathfrak{r}_{i} \approx \operatorname{Hom}_{B, \beta-1}\left({ }_{B} \mathfrak{l}_{i},{ }_{B} B\right) & (B \text {-right }) .
\end{array}
$$

Now, Kasch [10] studied scalar products of free modules over a certain type of ring, called $S$-ring; a ring $B$ is called an $S$-ring when it satisfies minimum condition and, moreover, the right annihilator $r(\mathfrak{l})$ in $B$ of a left ideal $\mathfrak{l}$ different from $B$ and the left annihilator $l(\mathfrak{r})$ in $B$ of a right ideal $\mathfrak{r}$ different from $B$ are always both different from 0 ;

$$
r(\mathfrak{l}) \neq 0, l(\mathfrak{r}) \neq 0 \quad(\mathfrak{l} \neq B, \mathfrak{r} \neq B) .
$$

(Kasch studied only ordinary 1-1-scalar products but a generalization to 1- $\beta$ (or $\beta_{0^{-}-\beta_{-}}$) case is rather immediate). We first observe that his proof to Satz 1 there remains valid for a case where the ambient spaces $\mathbb{Z}, \Re$ need not be vector spaces (i.e. free modules). So we have (changing "left" and "right" in Kasch's formulation)

Lemma. Let $\mathbb{L}, \mathfrak{R}$ be respectively a left-and a right module over an S-ring $B$, and let $\mathfrak{R}_{1}$ be a finitely generated (or, more generally, countably generated) free $B$-right submodule of $\Re$. Suppose that $\&$ and $\Re$ have a regular1- $\beta$-scalar product $\left\langle>\right.$ in $B$. Then $\&$ has a free B-left-submodule $\mathfrak{Q}_{1}$ such that $\mathfrak{L}_{1}$ and $\mathfrak{R}_{1}$ have free bases orthogonal with respect to \langle\rangle ; the $B$ (left-) rank of $\mathfrak{Q}_{1}$ is then equal to the $B$-(right) rank of $\Re_{1}$, where the B-rank of a free $B$-module is defined to be the number of terms in its B-free basis.

Using this lemma we can show that for an $S$-ring $B$ a converse of the above remark holds. Thus we have

PRoposition 15. Let $\mathfrak{R}$ be a finitely generated projective right:module over an S-ring $B$, and $\&$ a B-left-module. If $\mathfrak{Z}$ and $\mathfrak{R}$ have a regular $1-\beta$-scalar product $\langle>$ in $B$, then

1) $\mathfrak{L} \approx \operatorname{Hom}_{B, \beta}\left(\Re_{B}, B_{B}\right)$ (B-left), $\mathfrak{R} \approx \operatorname{Hom}_{B, \beta^{-1}}\left({ }_{B} \&,{ }_{B} B\right) \quad(B$-right $)$; 
2) for every projective B-right-submodule $\Re_{1}$ of $\Re$ we have $R\left(L\left(\Re_{1}\right)\right)=\Re_{1}$, where $L\left(\Re_{1}\right)$ (resp. $R\left(L\left(\Re_{1}\right)\right)$ is the left (resp. right) annihilator of $\Re_{1}$ (resp. $\left.L\left(\Re_{1}\right)\right)$ in $\mathfrak{Q}$ (resp. $\left.\mathfrak{R}\right)$ with respect to $\langle>$, and direct decompositions

$$
\mathfrak{L}=\mathfrak{Q}_{1} \oplus L\left(\Re_{1}\right), \quad \Re=\mathfrak{R}_{1} \oplus R\left(\mathfrak{Q}_{1}\right)
$$

hold with some B-left-submodule $\mathfrak{Q}_{1}$ of $\mathbb{Q}$.

Proof. We obtain a $B$-left-monomorphism $\mathfrak{Z} \rightarrow \operatorname{Hom}_{B, \beta}\left(\Re_{B}, B_{B}\right)$ by associating each element $y$ of $\mathfrak{Q}$ with the element $\varphi_{y}$ of $\operatorname{Hom}_{B, \beta}\left(\mathfrak{R}_{B}, B_{B}\right)$ such that $\varphi_{y} x$ $=\langle y, x\rangle$. Hence we may, and shall, consider $\mathbb{Q}$ as a submodule of $\operatorname{Hom}_{B, \beta}\left(\Re_{B}\right.$, $B_{B}$ ).

On the other hand, there is a finitely generated free $B$-right-module $\mathfrak{R}_{0}$ which has $\Re$ as a direct summand; $\Re_{0}=\Re \oplus \mathfrak{R}^{\prime}$. We have, as usual,

$$
\operatorname{Hom}_{B, \beta}\left(\Re_{0 B}, B_{B}\right)=\operatorname{Hom}_{B, \beta}\left(\Re_{B}, B_{B}\right) \oplus \operatorname{Hom}_{B, \beta}\left(\Re_{B}^{\prime}, B_{B}\right) \text {. }
$$

As the $B$-right-module $\Re^{\prime}$ is projective, $\operatorname{Hom}_{B, \beta}\left(\Re_{F}^{\prime}, B_{B}\right)$ and $\Re^{\prime}$ have a regular $1-\beta$-scalar product in $B$. Further, we are given a regular 1- $\beta$-scalar product, $\langle>$, for $\mathfrak{L}$ and $\mathfrak{R}$. From these two regular $1-\beta$-scalar products we obtain a regular $1-\beta$-scalar product in $B$, for $\mathfrak{i} \oplus \operatorname{Hom}_{B, \beta}\left(\mathfrak{R}_{l,}^{\prime}, B\right)$ and $\mathfrak{R} \oplus \mathfrak{R}^{\prime}$. Hence, by the above lemma, $\mathfrak{L} \oplus \operatorname{Hom}_{B, \beta}\left(\mathfrak{R}_{B}^{\prime}, B\right)$ has a submodule having a free basis orthogonal to a such of $\mathfrak{R} \oplus \mathfrak{R}^{\prime}=\mathfrak{R}_{0}$. Since $\mathfrak{L} \oplus \operatorname{Hom}_{B, \beta}\left(\mathfrak{R}_{B}^{\prime}, B\right)$ is contained in $\mathrm{H}_{\mathrm{Oom}, \beta}\left(\Re_{0 B}, B_{B}\right)$, by $(50)$, it follows that

(B-right-rank of $\left.\Re_{0}\right) \leqq\left(B\right.$-right-rank of $\left.\operatorname{Hom}_{B, 3}\left(\Re_{0 B}, B_{B}\right)\right)$,

and the equality holds only when $\mathfrak{Z}=\operatorname{Hom}_{B, \beta}\left(\Re_{B}, B_{B}\right)$. The $B$-left-rank of $\operatorname{Hom}_{B, \beta}\left(\Re_{0 B}, B_{B}\right)$ is, however, clearly equal to the $B$-right-rank of $\Re_{0}$. Hence $\&$ coincides necessarily with $\operatorname{Hom}_{B, \beta}\left(\mathfrak{N}_{B}, B_{B}\right)$, to have the first relation of the assertion 1) in our proposition. The second relation then follows by the general remark we made above.

Let next $\Re_{1}$ be any projective $B$-right-submodule of $\Re$. As our regular (1- $\beta$-) scalar product $\left\langle>\right.$ for $\mathfrak{L}, \mathfrak{R}$ induces a such for the residue-module $\mathbb{Q} / L\left(\mathfrak{R}_{1}\right)$ and $\mathfrak{R}_{1}$, the assertion 1 ) (applied to $\mathfrak{R}_{1}$ in place of $\Re$ ) shows that the $B$-left module $\mathfrak{L} / L\left(\Re_{1}\right)$ is projective. Hence there is a $B$-left-submodule $\mathfrak{Q}_{1}$ of $\mathfrak{Q}$ such that $\mathfrak{Q}=\mathfrak{Q}_{1} \oplus L\left(\mathfrak{R}_{1}\right)$. By our general remark we have then $\mathfrak{R}=R\left(\mathfrak{Q}_{1}\right) \oplus R\left(L\left(\mathfrak{R}_{1}\right)\right)$. We have also $\Re_{1} \approx \operatorname{Hom}_{B, \beta-1}\left({ }_{B}\left(\mathbb{L} / L\left(\Re_{1}\right)\right),{ }_{B} B\right)$ ( $B$-right). Since also the $B$-rightmodule $R\left(L\left(\Re_{1}\right)\right.$ ) is, as a direct summand of $\mathfrak{R}$, projective, we have similarly 
$R\left(L\left(\Re_{1}\right)\right) \approx \operatorname{Hom}_{P, \beta^{-1}}\left({ }_{B}\left(\mathcal{L} / L\left(R\left(L\left(\Re_{1}\right)\right)\right)\right),{ }_{B} B\right)$ (B-right). But here $L\left(R\left(L\left(\Re_{1}\right)\right)\right)$ $=L\left(\Re_{1}\right)$. Hence $\Re_{1} \approx R\left(L\left(\Re_{1}\right)\right)$ (B-right) and necessarily $\Re_{1}=R\left(L\left(\Re_{1}\right)\right)$ in fact. This shows $\mathfrak{R}=R\left(\Omega_{1}\right) \oplus \Re_{1}$.

Now, in case the ground ring $B$ is an S-ring our previous characterizations of Frobenius extensions by means of bilinear homomorphisms and scalar products, given in Proposition 4, Corollary 5 and a remark succeeding them, may be modified as follows :

Proposition 16. Let $A$ be a ring having an S-ring $B$ as a subring, and assume that the B-left-module $A$ is finitely generated and projective (i.e. the condition $1_{r}$ ) in Proposition 1). Let $\beta$ be an automorphism of $B$. Then $A$ is $\beta$ Frobenius over $B$ if and only if either one of the following conditions holds:

$\alpha)$ there is $a(B, 1)$ - $(B, \beta)$-homomorphism $\pi$ satisfying $\left.\left.\mathrm{i}_{r}\right), \mathrm{i}_{l}\right)$ of Proposition 4 (i.e. that $a=0(a \in A)$ is implied either by $\pi a A=0$ or by $\pi A a=0)$;

$\beta)$ there exists a regular 1- $\beta$-scalar product $\langle>$ in $B$ of the $B$-left-module $A$ and the $B$-right-module $A$ such that $\langle x, y z\rangle=\langle x y, z\rangle$ for all $x, y, z \in A$.

Proof. The equivalence of $\alpha$ ) and $\beta$ ) we noted already in $\S 1$ (in fact without the $S$-ring assumption). Also the necessity of the conditions $\alpha$ ), $\beta$ ) was seen in $\S 1$ (again without the $S$-ring assumption).

To prove the sufficiency, assume $\beta$ ). Then, in proving 1) of the preceding proposition we have seen that $a \rightarrow \varphi_{a}$ with $\varphi_{a} x=\langle a, x\rangle$ gives a $B$-left-isomorphism of $A$ (not only into but) onto $\operatorname{Hom}_{B, \beta}\left(A_{B}, B_{B}\right)$. In terms of the $(B, 1)$ ( $B, \beta$ )-homomorphism $\pi$ of ${ }_{B} A_{B}$ into $B$ given by $\pi x=\langle 1, x\rangle$, this means that the condition $\mathrm{ii}_{r}$ ) of Proposition 4 holds. $A$ is thus $\beta$-Frobenius over $B$ by Corollary 5 .

\section{REFERENCES}

[1] H. Cartan-S. Eilenberg, Homological Algebra, Princeton 1956.

[2] B. Eckmann-A. Schopf, Über injektive Moduln, Arch. Math. 4 (1953), 75-78.

[3] S. Eilenberg, Homological dimension and syzygies, Ann. Math. 64 (1956), 246-269.

[4] S. Eilenberg-T. Nakayama, On the dimension of modules and algebras II, Nagoya Math. J. 9 (1955), 1-16.

[5] N. S. Gopalakrishnan-N. Ramabhadran-R. Sridharan, A note on the dimension of modules and algebras, Jour. Indian Math. Soc. 21 (1957), 185-192.

[6] K. Hirata, On relative homological algebras of Frobenius extensions, Nagoya Math. J. 15 (1959), 17-28.

[ 7] G. Hochschild, Relative homological algebra, Trans. Amẹr. Math. Soc, 82 (1956), 246269 , 
[ 8 ] M. Ikeda, A characterization of quasi-Frobenius rings, Osaka Math. J. 4 (1952), 203210.

[ 9 ] J. P. Jans, Some remarks on symmetric and Frobenius algebras, Nagoya Math. J. 16 (1960), 65-71.

[10] F. Kasch, Grundlagen einer Theorie der Frobeniuserweiterungen, Math. Ann. 127 (1954), 453-474.

[11] H. Nagao-T. Nakayama, On the structure of (Mo)- and (Mu)-modules, Math. Z. 59 (1953), 164-170.

[12] T. Nakayama, On Frobenius algebras II, Ann. Math. 42 (1941), 1-21.

[13] T. Nakayama-T. Tsuzuku, A remark on Frobenius extensions and endomorphism rings, Nagoya Math. J. 15 (1959), 9-16.

\section{Mathematical Institute}

Nagoya University 